\section{Evidence summary: how can dietary advice to prevent dental erosion be effectively delivered in UK general dental practice?}

\author{
Original question submitted by Anh Lam, November 2009 \\ Reviewer: Chris Fox' \\ Information Scientist: Helen Nield
}

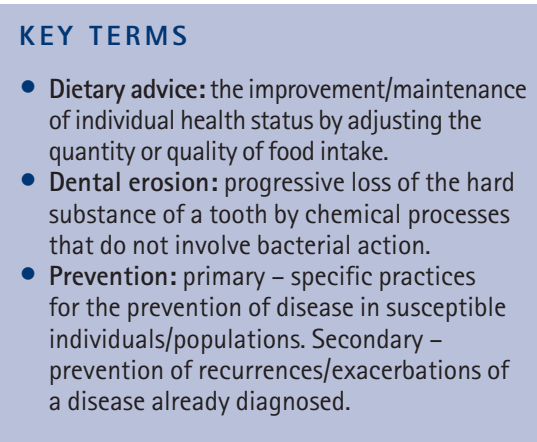

- Dietary advice: the improvement/maintenance of individual health status by adjusting the quantity or quality of food intake.

Dental erosion: progressive loss of the hard that do not involve bacterial action.

Prevention: primary - specific practices for the prevention of disease in susceptible prevention of recurrences/exacerbations of a disease already diagnosed.

Since August 2009, members of the newly redeveloped Primary Care Dentistry Research Forum (www.dentistryresearch. org) have taken part in an online vote to identify questions in day-to-day practice that they felt most needed to be answered with conclusive research. The question which received the most votes formed the subject of a critical appraisal of the relevant literature. Each month a new round of voting takes place to decide which further questions will be reviewed. Dental practitioners and dental care professionals are encouraged to take part in the voting and submit their own questions to be included in the vote by joining the website.

This paper details a summary of the findings of the third critical appraisal. In conclusion, the critical appraisal has identified that there is no conclusive answer to this question at present. No evidence was identified relating to the effectiveness of different strategies to apply dietary advice for the prevention of dental erosion. Given the apparently increasing prevalence of the disease in industrialised countries, this appears to be a priority area for future primary research.

\section{BACKGROUND}

In $2000,52-65 \%$ of children and adolescents were found to have tooth surface loss due to dental erosion. 'Erosive tooth wear has for many years been a condition of little interest to clinical dental practice or dental public health', although this perception is changing. ${ }^{2}$ Public recognition of dental erosion is poor.

The trend towards longer retention of dentition $^{3}$ is making tooth wear (whether dental erosion or other forms) an increasing clinical challenge. Progression of erosion is believed to be greater in older adults (52-56 years) compared to younger (32-36 years). ${ }^{4}$

Multifactorial approaches to prevention and control of dental erosion have been described ${ }^{5-7}$ in response to the spectrum of chemical, biological and

\footnotetext{
'PhD Student, Peninsula Dental School Correspondence to: Beth Caines, Shirley Glasstone Hughes Trust Fund Administrator, British Dental Association, 2 Caspian Point, Cardiff Bay, CF10 4D0 Email: b.caines@bda.org; Tel: +44 (0)29 20436184 Sponsored by the Shirley Glasstone Hughes Trust
}

DOI: 10.1038/sj.bdj.2010.206 behavioural influences upon the development of dental erosion. ${ }^{8}$ Diet plays an important role in the prevention of oral diseases. ${ }^{9,10}$ Dietary factors, particularly acidic food and drinks, contribute to the development of dental erosion.

The current review question specifically focuses upon how dietary advice can be effectively delivered in general dental practice.

\section{AIM}

This review aimed to identify, evaluate and summarise primary research studies which are either of:

- Interventions where the dietary advice given has been solely related to preventing dental erosion, or of

- Interventions where, in an overall broader campaign to improve/protect oral/dental health using dietary advice, the prevention (primary/ secondary) of dental erosion is a stated aim.

\section{REVIEW METHOD}

An initial search was made of Ovid MEDLINE (1950 to week 5, October 2009) using the search terms diet therapy, tooth erosion and prevention. Thirtyfour papers were identified and 34 were excluded (no interventions identified).

O'Sullivan E, Milosevic A. UK National Clinical Guidelines in Paediatric Dentistry: diagnosis, prevention and management of dental erosion. Int J Paediatr Dent 2008; 18(Suppl 1): 29-38.

Magalhaes A C, Wiegand A, Rios D, Honorio H M, Buzalaf M A R. Insights into preventive measures for dental erosion. J App/ Oral Sci 2009; 17: 75-86.

Note: The following review protocol has been published by The Cochrane Collaboration, and the authors have indicated their findings may be reported during 2010:

Ashcroft A, Harris R, Dailey Y. One-to-one dietary interventions undertaken in a dental setting for a change in dietary behaviour and the prevention of dental caries and erosion. Cochrane Database Syst Rev 2007; (2): CD006540. DOI: 10.1002/14651858.CD006540. 


\section{EVIDENCE SUMMARY}

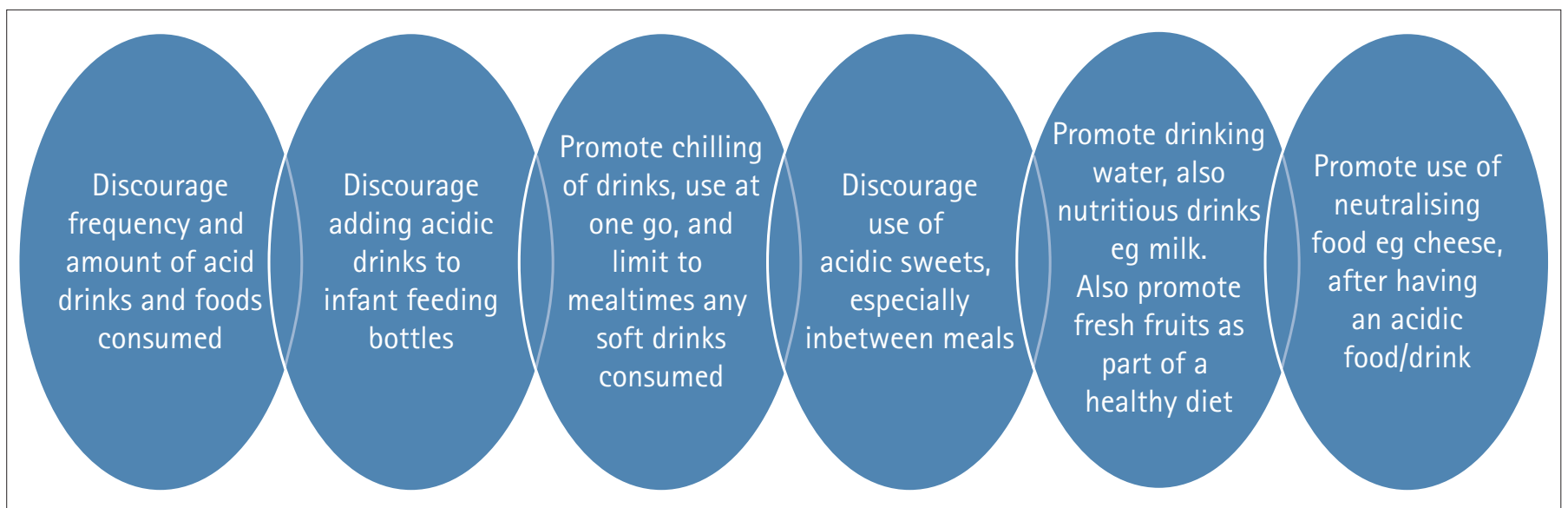

Fig. 1 Dietary recommendations in dental practice in relation to dental erosion ${ }^{11}$

A second search of Ovid MEDLINE (1950 to week 3, November 2009) was then undertaken using the search terms diet, tooth erosion and prevention. One hundred papers were identified and 100 excluded (no interventions identified).

Further searches included CEBD, Cochrane Oral Health Group, CRD, ADA and National Library for Public Health.

\section{FINDINGS}

We could find no studies that have compared alternative strategies for the delivery of dietary advice to prevent dental erosion. No studies were found either where the dietary advice was solely related to dental erosion or as a stated element of a broader oral/dental health campaign.
Whilst not the primary focus of this review, more evidence was identified about the dietary messages that may be communicated to prevent dental erosion. These messages and details of recent reviews/guidelines on the prevention of dental erosion, including through dietary means, are provided in Figure 1 and Table 1.

In summary, there was no evidence found on the relative effectiveness of different strategies to apply dietary advice for the prevention of dental erosion. Given the apparently increasing prevalence of the disease in industrialised countries, it appears to be a priority area for future primary research.

1. Department of Health. National diet and nutrition survey: young people aged 4 to 18 years. Volume 2 report of the oral health survey. London:
The Stationery Office, 2000

2. Lussi A. Erosive tooth wear - a multifactorial condition of growing concern and increasing knowledge. Monogr Oral Sci 2006; 20: 1-8.

3. Steele J. NHS dental services in England. An independent review led by Professor Jimmy Steele. London: Department of Health, 2009.

4. Lussi A, Jaeggi T, Zero D. The role of diet in the aetiology of dental erosion. Caries Res 2004; 38(Suppl 1): 34-44.

5. Amaechi B T, Higham S M. Dental erosion: possible approaches to prevention and control. J Dent 2005; 33: 243-252.

6. Imfeld T. Prevention of progression of dental erosion by professional and individual prophylactic measures. Eur J Oral Sci 1996; 104: 215-220.

7. Department of Health/British Association for the Study of Community Dentistry. Delivering better oral health: an evidence-based toolkit for prevention. 2nd ed. London: Department of Health, 2009

8. Lussi A Jaeggi T. Erosion - diagnosis and risk factors. Clin Oral Investig 2008; 12(Suppl 1): S5-S13.

9. Moynihan PJ. The role of diet and nutrition in the etiology and prevention of oral diseases. Bull World Health Organ 2005; 83: 694-699.

10. Moynihan P, Peterson P E. Diet, nutrition and the prevention of dental diseases. Public Health Nutr 2004; 7(1A): 201-226.

11. Auad S, Moynihan P. Diet and dental erosion. Quintessence Int 2007; 38: 130-133. 